\title{
Removal of severely encrusted forgotten ureteral stents by minimal access techniques
}

\author{
Neville D Perera ${ }^{1}$ and Manjula Wijewardena ${ }^{2}$ \\ (Index words: Extracorporeal shock wave lithotripsy, cystoscopic lithotrity)
}

\section{Introduction}

Forgotten JJ stents are cited as one of the major disadvantages when compared with percutaneous nephrostomy (PCN) for relieving obstructed kidneys. However, published literature is scarce regarding ways of removing them without open exploration. Two recent cases are reported where extracorporeal shock wave lithotripsy (ESWL) and endoscopic intracorporeal lithotrity were used to remove JJ stents.

\section{Case reports}

Case 1. A 35-year old woman who underwent a rightsided Culp-Scardino pyeloplasty 3 years before, was admitted with a complaint of difficulty in passing urine of 2 months' duration. She also had frequent low grade fever which settled with short courses of antibiotics.

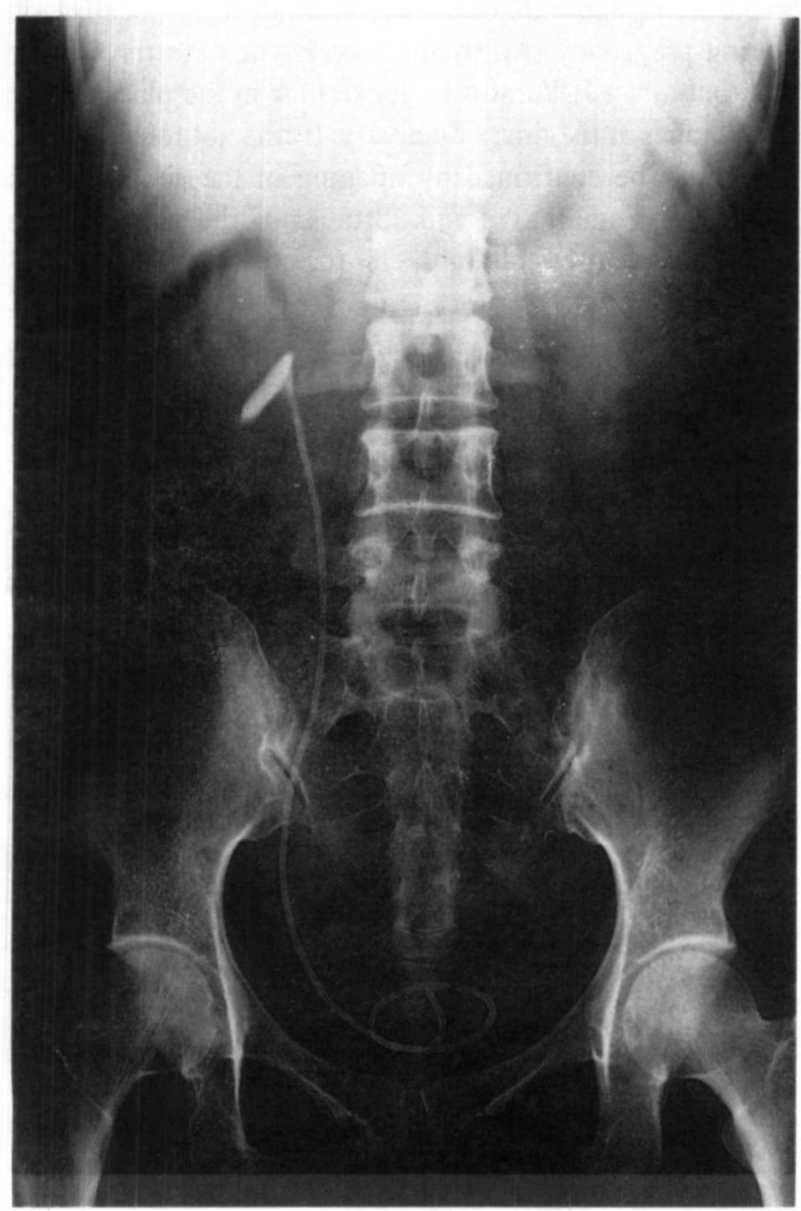

Figure. Plain radiograph showing the grossly encrusted JJ stent.
In the course of investigation a grossly encrusted forgotten JJ stent was seen in the xray (Figure). She was submitted to cystoscopic lithotrity to the bladder coil and $4 \times 8 \times 4000$ ESWL (Wolf piezolith-2500) pulses to the renal coil of the stent. Once there was fluoroscopic evidence of dispersion of upper encrustations the stent was removed by rigid cystoscopy using a grasper.

Case 2. A 42-year old man who underwent left-sided ureterolithotomy 9 months before was admitted for removal of an encrusted JJ stent by flexible cystocopy. This resulted in a fracture of the stent and removal of only a mid-portion of it. Using the ureterorenoscope with the pneumatic lithotripter, dislodgment of stent encrustations of the upper coil was achieved and the stent fragment was trapped in a dormia basket. After a session of ESWL $10 \times 4000$ to the renal coil, the dormia basket was pulled out by jerky movements under fluoroscopy, successfully delivering the fragmented segment. The removal of the encrusted bladder coil was achieved by cystoscopic lithotrity.

\section{Discussion}

Forgotten JJ stent is an uncommon complication occurring when the patient fails to appreciate the long term consequences of retaining a stent (1). It is more common in patients who tolerate stents well than in those who have discomfort. Submitting a patient to open exploration to deliver a non-yielding $\mathrm{JJ}$ stent defeats the very purpose of introducing it as a part of minimal access therapy. As there is no standard practice, many ingenious methods are used to remove such stents, depending on the circumstances in the individual patients $(1,2,3)$. The two cases described show that a combination of minimal access methods could be safely and successfully applied without resorting to formal exploration in most cases.

\section{References}

1. Shandera KC. Minimally invasive management of the calcified ureteral stent. Techniques in Urology 1997; 3: 54-7.

2. Borboroghe PG, Kane CJ. Current management of severly encrusted ureteral stents with a large associated stone burden. Journal of Urology 2000; 164: 648-50.

3. Gotwald TF, Peschel R, Frauscher L, Neururer R,Zzur-Nedden $\mathrm{D}$, et al. Indwelling ureteral stent fragmentation with severe encrustation and stone formation. Journal of Urology 1999; 162: 788.

'Urological Surgeon, and ${ }^{2}$ Senior Urological Registrar, Department of Urology, National Hospital of Sri Lanka, Colombo. (Received 13 August 2001, accepted 20 October 2001. Corresponding author NDP, telephone +941778061 ) 